\title{
Investigating of variations in BRCA1 gene in Iranian families with breast cancer
}

\author{
Amir Mehrgou ${ }^{1}$, Mansoureh Akouchekian*1, Simin Hemati ${ }^{2}$ \\ Received: 30 Mar 2018 \\ Published: 17 Sep 2018
}

\begin{abstract}
Background: Breast cancer is one of the most common cancers among Iranian women whose relationship with mutation status in BRCA1 is previously approved. Therefore, screening of the most mutated exons in BRCA1 in hereditary breast cancer patients provides beneficial information about the main disease-causing reason.

Methods: A total of 14 Iranian hereditary breast cancer patients participated in this case series study. DNA was extracted from patients' blood samples for PCR assay. The quality of PCR products was determined using horizontal electrophoresis. Then, sequencing and analysis of the sequencing results were performed to investigate variation status in the sequences.

Results: Five variants in 4 patients were found, including 1 pathogenic variant in exon 16 (H1686Q, NM_007294.3:c.5058T >A) and 4 novel intronic variants of uncertain significance (NC 000017.11:41228314G $>\mathrm{T}$, NC 000017.11:41228309C $>\mathrm{T}$, $\mathrm{NC} 000017.11: 41228317 \mathrm{G}>\mathrm{T}$, and $\mathrm{NC} 000017.11: 41203042 \mathrm{G}>\mathrm{A}$ ) in $\mathrm{BRCA} 1$. This study was the first to report 1 rare pathogenic variant in BRCA1 (H1686Q, NM_007294.3: c.5058T>A) in an Iranian family as the main disease-causing reason. Another interesting finding was non-existence of variations in almost all globally-reported and mutated exons in BRCA1.

Conclusion: Investigation of these exons in BRCA1 showed the uniqueness of mutation pattern in Iranian breast cancer patients compared to other world regions. Due to the existence of other BRCA1 exons and also other predisposing genes in breast cancer, the main cause of cancer development in other participants might have been put in those exons and genes. We concluded that the most mutated exons in BRCA1 in Iranian population may not be the same as those found in other parts of the world.
\end{abstract}

Keywords: Hereditary breast cancer, BRCA1 gene, Variation

Copyright $\odot$ Iran University of Medical Sciences

Cite this article as: Mehrgou A, Akouchekian M, Hemati S. Investigating of variations in BRCA1 gene in Iranian families with breast cancer. Med J Islam Repub Iran. 2018 (17 Sep);32:87. https://doi.org/10.14196/mjiri.32.87

\section{Introduction}

Breast cancer is a heterogeneous disease that includes several molecular subgroups (1).

The incidence rate of breast cancer varies in different geographical regions (2). According to Annual Iranian National Cancer Registration Report 2008-2009, breast cancer among Iranian women, has been registered as the second and first most common cancer, with the incidence rate of $11.31 \%$ and $24.86 \%$, respectively (3).

Two major factors, which cause developing breast cancer in the general population, are early menstruation and late menopause (4).

The existence of genetic-predisposing factors can lead to early-onset breast cancer at younger than 50 years (2). Among genetic causes, mutation in BRCA1 gene is

Corresponding author: Dr Mansoureh Akouchekian,akouchekian.m@iums.ac.ir

1. Department of Medical Genetics and Molecular Biology, School of Medicine, Iran University of Medical Sciences, Tehran, Iran.

2. Department of Oncology, Faculty of Medicine, Isfahan University of Medical Sciences, Isfahan, Iran. known as predisposing factor for breast cancer (1).

BRCA1 is located on chromosome $17 \mathrm{q}$, and any mutations or changes in this gene can lead to increased risk of breast and ovarian cancers $(2,3)$.

Germline mutation in the tumor suppressor gene BRCA1 is one of the main known risk factors for earlyonset breast cancer (5).

Also, there are some variations other than mutations, called variants of uncertain significance (VUS) and polymorphisms. VUSs in BRCA1 are a type of variants whose functions in protein level are yet to be survived. However, BRCA1 polymorphisms may affect protein, causing it to lose function, and thereby to increase the risk of developing breast cancer (6).

$\uparrow$ What is "already known” in this topic:

Alterations in BRCA1 gene could increase the risk of breast cancer.

$\rightarrow$ What this article adds:

Mutation pattern of BRCA1 gene in Iranian breast cancer patients could be unique compared to other populations. Also, some new and reported variations in BRCA1 gene have been found. 
The aim in the present study was to report a pathogenic variant and variants of uncertain significance in the most mutated exons in BRCA1 among Iranian breast cancer patients.

\section{Methods}

\section{Data}

Exons $11,13,16$, and 18 to 23 are considered the most mutated exons in BRCA1 $(2,7,8)$.

Precise evaluation of family pedigrees and medical histories was performed to select suitable families for this study (Table 1). Informed consent forms were taken from all 14 female patients. Inclusion criterion was that all probands had to be Iranian breast cancer patients who had at least one first- or second-degree relative who had developed breast or ovarian cancer regardless of age or disease onset (preferably under 50 yrs.). The exclusion criterion was developing various cancer types in patients' family members which potentiated them as syndromic cases.

\section{DNA isolation}

The DNA extraction process was done manually by GeNet Bio $^{\circledR}$ DNA extraction kit and its instruction. The quality of extracted DNA was investigated through horizontal electrophoresis on agarose gel $(1 \%)$, with a voltage of 94 volts for 20 minutes.

\section{Primer designing}

At first, the consensus sequence of human BRCA1 was searched in GRCh38.p10 version of Ensembl genome browser (http://ensembl.org/index.html). Then, all primers were designed by Primer 3 online software version 0.4.0 (http://bioinfo.ut.ee/primer3-0.4.0/primer3/input.htm), with observing ideal conditions including melting temperatures $(\mathrm{Tm})\left(56-63.5{ }^{\circ} \mathrm{C}\right)$, length of primers $(19-24$ bps) and their products (under $1000 \mathrm{bps}$ ), CG percentages $(40 \%-60 \%)$, the number of purine bases in $3^{\prime}$ end, etc. The primers were designed to ampilify a part of intronic sequences surrounding the target exons, so the possible intronic variants could be detected too. The primers were checked in and approved by PrimerBlast online software (https://ncbi.nlm.nih.gov/tools/primer-blast/index.cgi) and UCSC genome browser (in-silico PCR) (https://genome. ucsc.edu/cgi-bin/hgPcr) to avoid regions of cross homology and approve quality and specificity. Then, in the final step, Korean Macrogen ${ }^{\circledR}$ company was asked to synthesize these primers (Table 2).

\section{PCR set up}

PCR programs (Table 3) have been set up with a positive control sample and a patient sample.

\begin{tabular}{|c|c|c|c|}
\hline \multicolumn{2}{|l|}{ Characteristics } & Number & Frequency $(\%)$ \\
\hline \multicolumn{4}{|c|}{-Age of onset (year) } \\
\hline \multicolumn{2}{|l|}{$\leq 50$} & 9 & 64.3 \\
\hline \multicolumn{2}{|l|}{$>50$} & 5 & 35.7 \\
\hline \multicolumn{2}{|c|}{ The mean age at menarche (year) } & 14 & \\
\hline \multicolumn{2}{|c|}{ The mean age of first complete pregnancy (year) } & 22 & \\
\hline \multicolumn{4}{|c|}{-Laterality of disease } \\
\hline \multicolumn{2}{|l|}{ Left side } & 9 & 64.3 \\
\hline \multicolumn{2}{|l|}{ Right side } & 5 & 35.7 \\
\hline \multicolumn{4}{|c|}{-OCP* consumption } \\
\hline \multicolumn{2}{|l|}{ Positive } & 6 & 42.8 \\
\hline \multicolumn{2}{|l|}{ Negative } & 8 & 57.2 \\
\hline \multicolumn{4}{|c|}{-Diseased family members with breast/ovarian/prostate cancer } \\
\hline \multicolumn{2}{|l|}{ One } & 6 & 42.8 \\
\hline \multicolumn{2}{|l|}{ More than one } & 8 & 57.2 \\
\hline Exon number & Primer sequences & $\mathrm{Tm} .{ }^{\circ} \mathrm{C}$ & Product size \\
\hline \multirow[t]{2}{*}{11} & F: AATCCAGTCCTGCCAATGAG & 58.4 & $278 \mathrm{bps}$ \\
\hline & R: GAGATGATGTCAGCAAACCTAAGA & 61.8 & \\
\hline \multirow[t]{2}{*}{13} & F: TGTCTGTTGCATTGCTTGTG & 56.4 & $500 \mathrm{bps}$ \\
\hline & R: CCGCACATTTCTCATGTTGTA & 57.4 & \\
\hline \multirow[t]{2}{*}{16} & F: CTGAGCTGTGTGCTAGAGGTAA & 62.1 & $193 \mathrm{bps}$ \\
\hline & R: GTGGTTTTATGCAGCAGATGCAAG & 63.5 & \\
\hline \multirow[t]{2}{*}{18} & F: GGAAGGACCTCTCCTCTGTCA & 63.3 & $247 \mathrm{bps}$ \\
\hline & R: GTGCATTGATGGAAGGAAGCAA & 60.3 & \\
\hline \multirow[t]{2}{*}{19} & F: TTTTTGAGATGCGAGATGGAG & 57.4 & 804 bps \\
\hline & R: GACACCCCAGTGAAGTGAA & 57.3 & \\
\hline \multirow[t]{2}{*}{20} & F: CCTGGGAAGTAGCAGCAGAA & 60.5 & 299 bps \\
\hline & R: CCATCGTGGGATCTTGCTTA & 58.4 & \\
\hline \multirow[t]{2}{*}{21} & F: GCCTTCATCCGGAGAGTGTAG & 63.3 & $234 \mathrm{bps}$ \\
\hline & R: TCCATAGGGACTGACAGGTG & 60.5 & \\
\hline \multirow[t]{2}{*}{22} & F: AGTGACAGTTCCAGTAGTCCT & 59.4 & $185 \mathrm{bps}$ \\
\hline & R: AGTGATAAACCAAACCCATGCA & 58.4 & \\
\hline \multirow[t]{2}{*}{23} & F: AGGACCCTGGAGTCGATTG & 59.5 & 372 bps \\
\hline & R: GACCCTTGCATAGCCAGAAG & 60.5 & \\
\hline
\end{tabular}




\begin{tabular}{cc} 
Table 3. PCR setup programs in this study. \\
\hline PCR assay steps \\
\hline Denaturing & Temperature $\left({ }^{\circ} \mathrm{C}\right.$ ) and Time (Min) \\
\hline Denaturing in the cycle & $95 \& 5$ \\
Annealing & $94 \& 1$ \\
Exon 11 & \\
Exon 13 & $58 \& 1$ \\
Exon 16 & $58 \& 1$ \\
Exon 18 & $58 \& 1$ \\
Exon 19 & $57.5 \& 1$ \\
Exon 20 & $60 \& 1$ \\
Exon 21 & $57 \& 1$ \\
Exon 22 & $58 \& 1$ \\
Exon 23 & $57 \& 1$ \\
Extension in the cycle & $60 \& 1$ \\
Final Extension & $72 \& 1$ \\
& $72 \& 10$ \\
Cycle number & Exon $18: 30$ \\
\end{tabular}

This table shows all of temperatures and times used in this project's PCR programs. All of these PCR setup programs achieved by setting temperature gradient conditions for all exons' annealing step in Biorad Thermal Cycler ${ }^{\mathbb{B}}$ model T100.

\section{Sanger sequencing}

All PCR products were sent to the expert company to be sequenced by Sanger sequencing method and with ABI3500 sequencer machine.

\section{Sequence analysis}

The sequences were analyzed by Sequencher software version 5.3 and BLAST (Basic Local Alignment Search Tool). The novelty of all probable variants was checked by HGMD (http://hgmd.cf.ac.uk/ac/index.php) and Mutation Taster websites (http://mutationtaster.org), ExAC (http://exac.broadinstitute.org), and 1000 Genomes Browsers (http://internationalgenome.org/1000-genomesbrowsers) databases. Also, the pathogenicity of the variants was estimated by CADD Phred score (http://cadd.gs.washington.edu/score). All suspected sequences regarding the existence of novel pathogenic or likely pathogenic variants were rechecked and resequenced by reverse primers. Also, the pathogenicity of variants which led to change in amino acid sequence, was reconfirmed by Polyphen-2 prediction software (http://genetics.bwh.harvard.edu/pph2). The frequencies of found variants were checked in ExAC, 1000 Genomes, and GenomAD browsers (http://gnomad. broadinstitute.org) databases for globally-reported variants and were also investigated in Iranome database (http://iranome.ir) for variants reported in different Iranian ethnic groups.

\section{Results}

We analyzed 14 Iranian female breast cancer patients who had a family history of breast cancer to find any variations in 9 exons and their near intronic regions of BRCA1. Patients' blood samples were used to perform DNA extraction according to kit instruction. Exons 11, 13, 16 , and 18 to 23 of BRCA1 were selected due to redundant reports on their high potential to be mutated. Then, efficient primer pairs were designed and a specific PCR program was set up for each exon. All patients' DNA samples were sequenced by $\mathrm{ABI} 3500$ sequencer machine and then their results were analyzed by Sequencher software version 5.3 and BLAST database (http://blast.ncbi. nlm.nih.gov/Blast.cgi). Types of all found variants in this project have been classified by prediction sites, such as Mutation Taster and Cancer Genome Interpreter websites (http://cancergenomeinterpreter. org/home). As demonstrated in Table 4, in this case series study, 1 rare missense and pathogenic variant (H1686Q, NM_007294.3 (BRCA1:c.5058T $>$ A) in exon 16 (9) and 4 novel intronic variants of uncertain significance (NC_000017.11: $41228314 \mathrm{G}>\mathrm{T}, \quad \mathrm{NC} 000017.11: 41228309 \mathrm{C}>\mathrm{T}, \quad \mathrm{NC}$ 000017.11:41228317 $\overline{\mathrm{G}}>\mathrm{T}, \mathrm{NC} 000017.11: 41203042 \mathrm{G}>$ A) in BRCA1 were found (Fig. 1). A rare pathogenic variant (H1686Q, NM_007294.3 (BRCA1):c.5058T>A), which was predicted by PolyPhen-2 software as a possible damaging variant with a high score (2.696) (9), was found in this study for the first time in Iranian breast cancer population and for the second time in the world. The

Table 4. Variations reported in this study.

\begin{tabular}{|c|c|c|c|c|}
\hline $\begin{array}{l}\text { Location of } \\
\text { variation }\end{array}$ & Sequence variant & $\begin{array}{l}\text { Type of variation based } \\
\text { on Mutation Taster }\end{array}$ & $\begin{array}{l}\text { Type of variation based on CGI } \\
\text { (Cancer Genome Interpreter) }\end{array}$ & $\begin{array}{l}\text { CADD Phred } \\
\text { score }\end{array}$ \\
\hline Intron 13 & $\mathrm{NC} 000017.11: 41228314 \mathrm{G}>\mathrm{T}$ & Polymorphism & Not-protein affecting & 0.782 \\
\hline Intron 13 & $\mathrm{NC}_{0} 000017.11: 41228309 \mathrm{C}>\mathrm{T}$ & Polymorphism & Not-protein affecting & 1.457 \\
\hline Intron 13 & $\mathrm{NC}-000017.11: 41228317 \mathrm{G}>\mathrm{T}$ & Polymorphism & Not-protein affecting & 2.032 \\
\hline Exon $16(9)$ & H1686̄, NM_007294.3:c.5058T $>$ A & Disease-causing & Tier 1 & 25.7 \\
\hline Intron 20 & NC $000017.11: 41203042 \mathrm{G}>\mathrm{A}$ & Polymorphism & Not-protein affecting & 5.879 \\
\hline
\end{tabular}

This table shows location of found variants in BRCA1 gene, sequence variants names based on HGVS nomenclature. Mutation Taster detected found VUSs as polymorphisms but CGI website described them as variants which not affected on BRCA1 protein. CGI website (https://cancergenomeinterpreter.org/home) assumed the found pathogenic variant as Tier 1 to describe it as a variant with strong clinical significance. CADD Phred scores demonstrate the importance level of pathogenicity of the variants. 


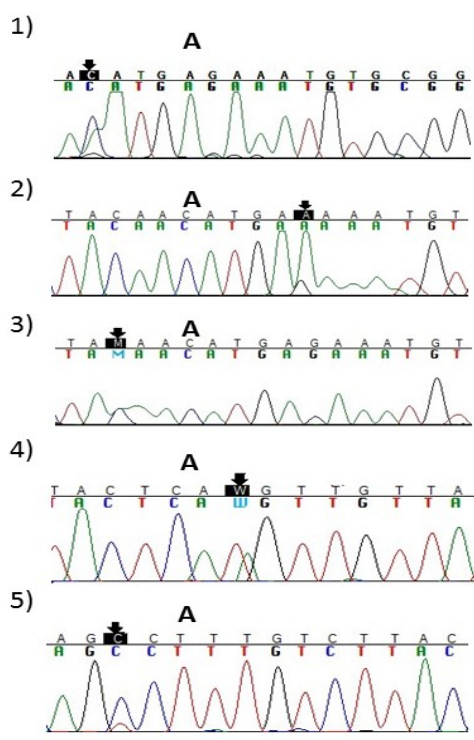

B
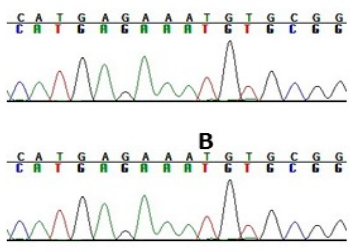

B

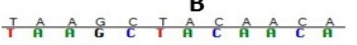

MnMmm

B

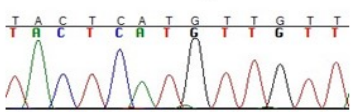

B

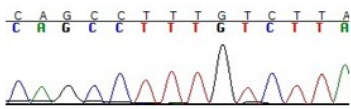

C

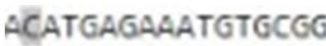

ACATGAGáatGTG

\#.............

141702

C

CATGAAAAATGTGC

....G......

141700

C

AAGCTAÄAACATGAGAAATGTGCGG

..................

141702

ACTCAAGTTGTTATGAAAACAGGTATACCAAGAACCT

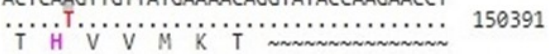

C

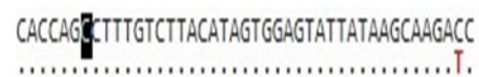

Fig. 1. (1) (A) Chromatogram of NC_000017.11:41228314G $>$ T variant in heterozygous state presents in one of breast cancer patients with 2 firstdegree and 1 second-degree relatives, with breast cancer and 1 third-degree relative with ovarian cancer. (B) Homozygous wild type allele for the variant. (C) The BLAST result for the variant. The highlighted region shows altered base in the sequence. (2) (A) Chromatogram of NC 000017.11:41228309C $>$ T variant in heterozygous state presents in one of breast cancer patients with 1 second-degree relative with breast cancer, 1 second-degree relative with prostate cancer and 3 more distant relatives with breast cancer. (B) Homozygous wild type allele for the variant. (C) The BLAST result for the variant. The highlighted region shows altered base in the sequence. (3) (A) Chromatogram of NC 000017.11:41228317G $>$ T variant in heterozygous state presents in one of breast cancer patients with 1 first-degree relative with ovarian cancer. (B) Homozygous wild type allele for the variant. (C) The BLAST result for the variant. The highlighted region shows altered base in the sequence. (4) (A) Chromatogram of H1686Q, NM 007294.3: c.5058T >A rare pathogenic variant in heterozygous state presents in one of breast cancer patients with 1 first-degree relative with early-onset breast cancer. (B) Homozygous wild type allele for the variant. (C) The BLAST result for the variant. The highlighted region shows altered base in the sequence. (5) (A) Chromatogram of NC_000017.11:41203042G $>$ A variant in heterozygous state presents in same patient noted as number 2. (B) Homozygous wild type allele for the variant. (C) The BLAST result for the variant. The highlighted region shows altered base in the sequence.

CADD Phred scores and other above-mentioned prediction results did not approve the relationship between the found intronic variants and breast cancer.

In the meanwhile, no variants were found in exons 11 , 13 , and 18 to 23 in BRCA1 in the patients.

\section{Discussion}

According to previous studies, breast cancer can be originated from both genetic and environmental factors. Environmental factors, which include the age at menarche and menopause, the age of first complete pregnancy, etc., constitute a large part of predisposition to this disease (10). However, genetic factors have a profound effect on predisposition to develop breast cancer.

BRCA1 and BRCA2 associated breast cancers constitute $25 \%$ to $30 \%$ of familial breast cancers and $3 \%$ of all types of breast cancers (11). Thus, these genes are considered as 2 major genetic factors predisposing to breast cancer.

To select suitable cases, almost all patients' medical histories were considered.

Most important finding in the present study, NM_007294.3: c.5058T>A (HGMD ID: CM087023), is a missense, loss of function and pathogenic variant, which was reported for the first time in Iran and for the second time worldwide in this study. This variant has been first found in an Italian family by Giannini et al. in 2008 (9) and caused histidine amino acid in position number of 1686 in BRCA1 protein to change to glutamine amino acid (H1686Q). In accordance with ExAC, 1000 Genomes, GenomAD browsers, and Iranome databases, this is a rare variant. This variant was found (in the heterozygous state) in a 46- years old patient who had one 49- year old sister with breast cancer. Considering the DNA sample that was extracted from the patient's blood and also considering the databases (mentioned before), this variant is a germline coding sequence variant, which led to early-onset breast cancer. The early onset of the disease in the proband's sister demonstrates that this variant might be responsible for disease development in both of them. However, due to non-availability of the patient's family members, we could not attain more details about this variant in her family. Also, the number of families carrying such rare variants is limited.

The age at menarche in this patient was less than 11 years old, and with respect to the mean age at menarche in our patients, she had an early menarche which had an inverse direct effect on breast cancer risk (12) and potentiated developing early-onset breast cancer.

According to investigated resources, the found VUSs were not responsible for developing or predisposing breast cancer.

BRCA1 protein plays as tumor suppressor in cells. Thus, the found pathogenic variant leads to production of a defective BRCA1 protein causes proliferating in breast tumor. On the other hand, mutated BRCA1 is not able to inhibit cell growth and reduction of BRCA1 dosage substantially even in heterozygous carrier has a negative 
effect on DNA repair process which results in aggregation of more mutations in the genome over time. Thereby, the root cause of disease development was identified in 1 patient.

Despite our anticipations, the number of variants found in this study was not equal to the expected number. These results suggest that there is a different mutation pattern regarding the most mutated exons in BRCA1 in Iranian families with hereditary breast cancer. Nevertheless, to recognize the pattern, more researches are required in the future.

There are several genes other than BRCA1 for predisposing to breast cancer, including BRCA2, CHEK2, PALB2, ATM, p53, PTEN, STK11, and CDH1. Thus, mutation in these genes might be the main reason for developing breast cancer in other participants.

Genetic testing on BRCA1 creates valuable information for detecting predisposed women to breast and ovarian cancer patients (13-16).

Complete resequencing of BRCA1 and BRCA2 genes is suggested for women with familial history of breast and/or ovarian cancer (17). However, this suggestion is practically expensive.

To decrease the cost, the screening should begin with the most mutated exons (according to their own geographical pattern of mutation) in BRCA1 $(15,18)$.

This study was a sparkle in introducing a new insight in screening and detection of main pathogenic variations according to geographical-specific mutation pattern in Iranian breast cancer families. However, the study's limitation was the small number of patients who were suitable to recruit. Thus, performing further studies on a large-sized group of Iranian hereditary breast cancer patients is necessary to further confirm our conclusions.

\section{Conclusion}

We reported for the first time a rare germline missense and loss of function pathogenic variant in BRCA1 in Iranian breast cancer population. The main reason for early-onset disease was recognized in 1 patient in this study. Conversely, the found VUSs did not increase the risk of breast cancer. According to the results of other participants, we concluded that the most mutated exons in BRCA1 in Iranian population may not be the same as those found in other parts of the world. Also, the root cause of disease in other patients may be located in other exons in BRCA1 or in some other predisposing genes to breast cancer. The results of this project need to be approved further through performing similar studies on a larger group of Iranian breast cancer families in future.

\section{Acknowledgements}

We appreciate Iran University of Medical Sciences for ethical approval and financial support. This work was supported by a grant from Iran University of Medical Sciences (project number: 26054).

\section{Conflict of Interests}

The authors declare that they have no competing interests.

\section{References}

1. Amin R, Morita-Fujimura Y, Tawarayama H, Semba K, Chiba N, Fukumoto M, et al. DeltaNp63alpha induces quiescence and downregulates the BRCA1 pathway in estrogen receptor-positive luminal breast cancer cell line MCF7 but not in other breast cancer cell lines. Mol Oncol. 2016;10(4):575-93.

2. Mehrgou A, Akouchekian M. The importance of BRCA1 and BRCA2 genes mutations in breast cancer development. Med J Islam Repub Iran. 2016;30:369.

3.Sadr-Nabavi A, Dastpak M, Homaei-Shandiz F, Bahrami AR, Bidkhori HR, Raeesolmohaddeseen M. Analysis of novel mutations in BRCA1 in Iranian families with breast cancer. HEREDITAS. 2014;151(2-3):38-42

4. Lecarpentier J, Nogues C, Mouret-Fourme E, Buecher B, GauthierVillars M, Stoppa-Lyonnet D, et al. Breast Cancer Risk Associated with Estrogen Exposure and Truncating Mutation Location in BRCA1/2 Carriers. Cancer Epidemiol. Biomarkers Prev. 2015;24(4):698-707.

5. Curtit E, Benhamo V, Gruel N, Popova T, Manie E, Cottu P, et al. First description of a sporadic breast cancer in a woman with BRCA1 germline mutation. Oncotarget. 2015;6(34):35616-24.

6. Jaure O, Alonso EN, Braico DA, Nieto A, Orozco M, Morelli C, et al. BRCA1 polymorphism in breast cancer patients from Argentina. Oncol. Lett. 2015;9(2):845-50.

7. Meng J, Shi YR, Niu RF, Fu L. Relationship between mutation of BRCA1 and susceptibility to early onset of breast cancer. Zhonghua yi xue za zhi. 2009;89(2):79-82.

8. Tazzite A, Jouhadi H, Nadifi S, Aretini P, Falaschi E, Collavoli A, et al. BRCA1 and BRCA2 germline mutations in Moroccan breast/ovarian cancer families: novel mutations and unclassified variants. Gynecol. Oncol. 2012;125(3):687-92.

9. Giannini G, Capalbo C, Ottini L, Buffone A, De Marchis L, Margaria E, et al. Clinical classification of BRCA1 DNA missense variants: H1686Q is a novel pathogenic mutation occurring in the ontogenetically invariant THV motif of the N-terminal BRCT domain J. Clin. Oncol. 2008;26(25):4212-4.

10. Ling H, Li S, Wu Y, Zheng YZ, Qiao F, Yao L, et al. Genetic evaluation of BRCA1 associated a complex genes with triple-negative breast cancer susceptibility in Chinese women. Oncotarget. 2016;7(9):9759-72

11. van den Broek AJ, Schmidt MK, van 't Veer LJ, Tollenaar RA, van Leeuwen FE. Worse breast cancer prognosis of BRCA1/BRCA2 mutation carriers: what's the evidence? A systematic review with meta-analysis. PloS one. 2015;10(3):e0120189.

12. Burgess S, Thompson DJ, Rees JMB, Day FR, Perry JR, Ong KK. Dissecting Causal Pathways Using Mendelian Randomization with Summarized Genetic Data: Application to Age at Menarche and Risk of Breast Cancer. Genetics. 2017;207(2):481-7.

13. Gambino G, Tancredi M, Falaschi E, Aretini P, Caligo MA. Characterization of three alternative transcripts of the BRCA1 gene in patients with breast cancer and a family history of breast and/or ovarian cancer who tested negative for pathogenic mutations. Int. J. Mol. Med. 2015;35(4):950-6.

14. Sanz DJ, Acedo A, Infante M, Duran M, Perez-Cabornero L, Esteban-Cardenosa E, et al. A high proportion of DNA variants of BRCA1 and BRCA2 is associated with aberrant splicing in breast/ovarian cancer patients. Clin. Cancer Res.2010;16(6):1957-67.

15. Abugattas J, Llacuachaqui M, Allende YS, Velasquez AA, Velarde $\mathrm{R}$, Cotrina J, et al. Prevalence of BRCA1 and BRCA2 mutations in unselected breast cancer patients from Peru. Clin. Genet. 2015;88(4):371-5.

16. Tutt A, Robson M, Garber JE, Domchek SM, Audeh MW, Weitzel $\mathrm{JN}$, et al. Oral poly(ADP-ribose) polymerase inhibitor olaparib in patients with BRCA1 or BRCA2 mutations and advanced breast cancer: a proof-of-concept trial. Lancet. 2010;376(9737):235-44.

17. Dombernowsky SL, Weischer M, Freiberg JJ, Bojesen SE, TybjaergHansen A, Nordestgaard BG. Missense polymorphisms in BRCA1 and BRCA2 and risk of breast and ovarian cancer. Cancer Epidemiol. Biomarkers Prev.2009;18(8):2339-42.

18. Narod SA. Screening for BRCA1 and BRCA2 mutations in breast cancer patients from Mexico: the public health perspective. Salud Publica Mex. 2009;51 Suppl 2:s191-6. 